SULFATIDE BILAYERS AS A SURFACE FOR CONTACT ACTIVATION IN HUMAN PLASMA

$$
\text { G. Tans }{ }^{\star} \text {, A.J. Verklel jł , } \mathrm{J} \mathrm{Yu}^{+} \text {, and J.H. Griffin }{ }^{+}
$$

*Dpt. Blochemistry, University of Limburg, Biomedical Centre, P.0.Box 616,

$6200 \mathrm{MD}$ Mastricht, The Netherlands
fInstitute of Molecular Blology, University of Utrecht, "De Uithof", Padualaan 8, 3584 CH Utrecht, The Netherlands

+Scripps Clinic and Research Foundation, Department of Immunology, 10666 North Torrey Pines Road, La Jo1la, California 92037

Received November 10,1987

Using a combination of gel permeation chromatography and freeze fracture electron microscopy it was found that the ability of sulfatides to promote contact activation in human plasma is associated with the ability of sulfatides to form bilayer structures. When sulfatides were dispersed in buffer at $70^{\circ} \mathrm{C}$ by vortexing, large liposomes were found ranging in size between 2-5 um diameter. Contact activation promoting activity was assoclated with these particles. Sonication resulted in the formation of small vesicles and stacked vesicles of 400-2000 A diameter and biological activity was associated with these vesicles. Homogeneous preparations of small unilamellar vesicles ( 500 A diameter) can be obtained by chromatography over Sepharose 2B. 1987 Academic Press, Inc.

When human plasma is incubated with cerebroside sulfates (sulfatides) contact activation occurs $[1,2]$ thereby initiating intrinsic blood coagulation, fibrinolysis, and the kinin-forming pathway [3]. Sulfatides are present in mammalian tissues [4] and because of their 1ipid-1ike nature they probably exist in solution in a form whose surface structure is 1sotropic thus offering an attractive model system with which to study the reactions Involved in contact activation.

Sulfatides, like kaolin [3] promote multiple proteolytic activations of the proteins of the contact system. Using purified bovine clotting factors sulfatides were shown to stimulate Factor XI activation by $\alpha$-Factor XII $[1,5]$ as well as activation of Factor XII by kallikrein, Factor $\mathrm{XI}_{\mathrm{a}}$ or plasmin [1]. Recently, we have shown that sulfatides strongly support

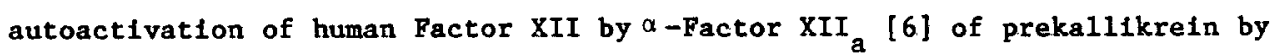
kallikrein [7] as well as the activation of Factor XII by kallikrein [8].

With more data becoming avallable on the interaction of contact factors with sulfatides it becomes increasingly more important to gain insight in the nature of the surface provided by sulfatides. 


\section{MATERIALS AND METIIODS}

Sepharose 2B was obtalned from Pharmacia, Uppsala, Sweden. Rabbit brain cephalin and bovine serum albumin were from Sigma Chemical, St. Louis, Mo. Azure-A, chloroform and methanol were from Aldrich Chemicals. Sulfatides from bovine brain were purchased from Supelco Inc., Bellefonte, Pa. High performancy thin layer chromatography plates, silicagel $60 \mathrm{~F}-254$ were from Merck. All reagents used were of the highest grade commercially available.

Procoagulant activitles of sulfatides were determined by their ability to shorten the clotting time in an activated partial thromboplastin time (aPTT) test in normal human plasma. $50 \mu 1$ Normal human plasma was added to $50 \mathrm{\mu l}$ cephalin (see below) and $100 \mu 1$ sulfatides (prepared as described below) were added. After incubation at $37{ }^{\circ} \mathrm{C}$ for 5 min, clotting was started by the addition of $50 \mu 1$ of a $50 \mathrm{mM} \mathrm{CaCl}{ }_{2}$ solution and the clotting time was measured.

Sulfatides were prepared by drying $5 \mathrm{ml}$ of a $2 \mathrm{mM}$ solution of sulfatides in chloroform/methanol $(1 / 1 ; v / v)$ under a stream of nitrogen and by subsequently resuspending the sulfatides in $2 \mathrm{ml} 50 \mathrm{mM}$ HEPES, $75 \mathrm{mM} \mathrm{NaCl}$ at $\mathrm{pH}$ 7.4 by vortexing at $70{ }^{\circ} \mathrm{C}$. When appropriate, the suspension was further sonicated for $5 \mathrm{~min}$ at $70{ }^{\circ} \mathrm{C}$ using a model W-375 sonicator from Heat Systems Inc at a $60 \%$ pulse at setting 2. After sonication the preparation was centrifuged for $5 \mathrm{~min}$ in an Eppendorf microfuge to remove possible contaminating pieces originating from the sonicator probe. No change in the 1ipid was detected after sonication as judged by thin layer chromatography on sillcagel $60 \mathrm{~F}-254$ (Merck) with chloroform/methano1/28\%ammonia (65/25/5; $\mathrm{v} / \mathrm{v} / \mathrm{v}$ ) as solvent. Sulfatide concentrations were determined according to Kean [9].

Cephalin was prepared by reconstituting the lipid with $\operatorname{Tris}$ buffered saline (10 mM Tris, $150 \mathrm{mM} \mathrm{NaCl}, \mathrm{pH} 7.4$ ) as indicated on the vial and was diluted tenfold before use in the coagulation assay.

Samples for freeze fracture were concentrated by centrifugation for 10 min at $100,000 \times \mathrm{xg}$ in a Beckman TL 100 ultracentrifuge. The samples were plpetted onto $3 \mathrm{~mm}$ gold planchets and frozen in liquld freon cooled by liquid nitrogen. Subsequently the samples were freeze fractured in a Balzers apparatus (Balzers High Vacuum Corp., Santa Ana, Ca.) at $-110{ }^{\circ} \mathrm{C}$ without etching [10]. Pt-C anodes, heated by electron bombardment from Tungsten cathodes, were used to cast replicas followed with a pure carbon reinforcement [11]. Replicas were cleaned in household bleach, mounted on bare 400 mesh grids and examined in a Hitachi $12 \mathrm{~A}$ electron microscope. Replicas were scanned and areas to be photographed were selected solely on the basis of sample concentration and replica quality.

\section{RESULTS AND DISCUSSION}

Sulfatides can be dispersed in an aqueous solution by vigorous vortexing at $70{ }^{\circ} \mathrm{C}$. When this is done an apparenty homogeneous solution is obtained which has a high turbidity and which is very potent when tested for contact promoting activity in normal human plasma using an activated partial thromboplastin time test (aPTT) [2]. To gain more insight in whether or not this procoagulant activity was associated with large particles or with the monomeric form of sulfatides which might be present in aqueous solutions, the preparation was chromatographed over sepharose $2 \mathrm{~B}$ and the eluting fractions were tested for the presence of sulfatides and for their ability to promote contact activation. Fig. 1 shows the result obtained. More than 90\% of the lipld appeared in the vold volume (Fig.1A) indicating the presence of large aggregates or particles of sulfatides. All vold volume fractions proved procoagulant with each fraction showing an optimal concentra- 

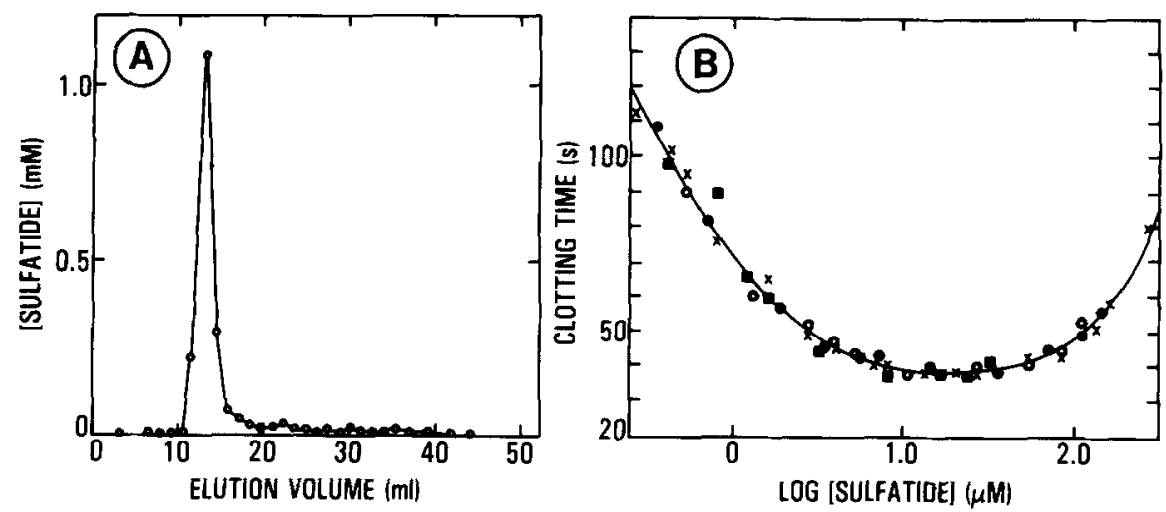

Figure 1. Elution profile and procoagulant activity of vortexed sulfatides chromatographed over Sepharose $2 \mathrm{~B}$

$0.75 \mathrm{ml}$ of a $5 \mathrm{mM}$ solution of vortexed sulfatides were passed over Sepharose $2 \mathrm{~B}(0.9 \times 30 \mathrm{~cm})$ at a flow rate of $5 \mathrm{ml} / \mathrm{hr}$ at room temperature. Fractions were collected (Panel $A$ ) and tested for their ability to shorten the clotting time in an aPTT test as described in Materials and Methods. Panel $B$ shows the clotting times as a function of the logarithm of the sulfatide concentration (expressed in MM) present. The various symbols represent the dose-response curves determined for fractions eluted at: 12 $\mathrm{ml}, 0-0 ; 13.5 \mathrm{ml}, x-x ; 15 \mathrm{ml}, 0-0$; and at $16.5 \mathrm{ml}, \mathbf{0}-\mathbf{a}$.

tion ranging from 7-40 $\mathrm{MM}(0.85-1.6$ for the logarithm of the sulfatide concentration) and optimal clotting times of 38 to $40 \mathrm{~s}$ (Fig.1B). Fractions eluting later in the column showed minor amounts of sulfatides and procoagulant activities proportional to these small amounts of sulfatides. The results presented in Figure 1 show that the procoagulant effect of vortexed sulfatides is associated with large particles.

Smaller particles were obtained by mild sonication of the vortexed sulfatide preparations. This resulted in a substantial decrease in turbidity and in a change of the gel filtration column profile as shown in Fig. 2A. In a typical run $10-20 \%$ of the lipid appeared in the vold volume representing a fraction of residual large particles. The main peak of material (approximately $60 \%$ of the total) eluted at a volume of 20-26 ml whereas the total internal volume was determined to be $34 \mathrm{ml}$. As can be seen from Fig. 2B, all fractions throughout the elution volume showed procoagulant activity. Optimal concentrations were slightly shifted as compared to vortexed sulfatides and ranged from 3-25 uM sulfatides present ( $0.48-1.4$ for the logarithm of the sulfatide concentration). Optima1 clotting t1mes ranged fron $38-44 \mathrm{~s}$.

It 18 well established that the general structure of lipid-containing preparations may withstand treatments involved in the freeze-fracture technique $[12,13]$. We, therefore, carried out freeze fracture electron microscopy studies of sulfatides in an aqueous environment. Fig. 3A shows a typical fracture face obtained from vortexed sulfatides. They appeared present as spherical liposomes with extended fracture surfaces as expected 

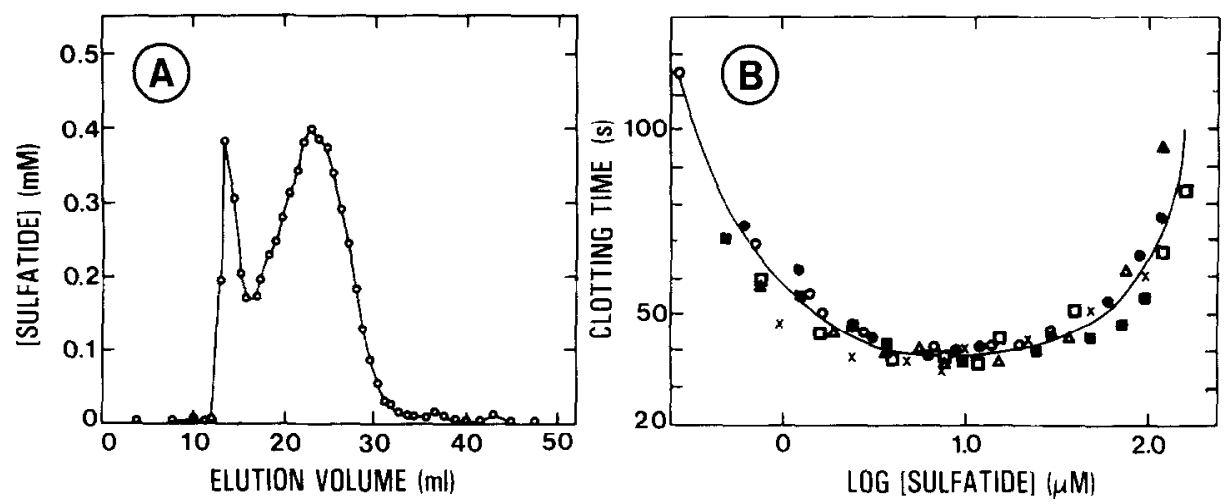

Figure 2. Elution profile and procoagulant activity of sonicated sulfatides chromatographed over Sepharose $2 B$

$1.5 \mathrm{ml}$ of a $5 \mathrm{mM}$ solution of sonicated sulfatides were passed over Sepharose 2B $(0.9 \times 50 \mathrm{~cm})$ at a flow rate of $5 \mathrm{ml} / \mathrm{hr}$ at room temperature. fractions were collected (panel A) and tested for procoagulant activity in an aPTT test as described under Materials and Methods. Panel $B$ shows the clotting times as a function of the logarithm of the sulfatide concentration (expressed in MM) present. The varlous symbols represent the doseresponse curves determined for fractions eluted at: $14 \mathrm{ml}, \Delta-\Delta ; 17 \mathrm{ml}, \mathbf{n}-\mathbf{\square}$ ; $20.5 \mathrm{ml}, . \square-\square ; 23.5 \mathrm{ml}, x-x ; 27 \mathrm{ml},--0$ and at $30 \mathrm{ml}, 0-0$.

for lamellar structures. None of the features unique for elther hexagonal or cubic phases were observed. The size distribution for vortexed sulfatide lfposomes ranged between 2-5 $\mu$ diameter. The fracture faces of the lipid
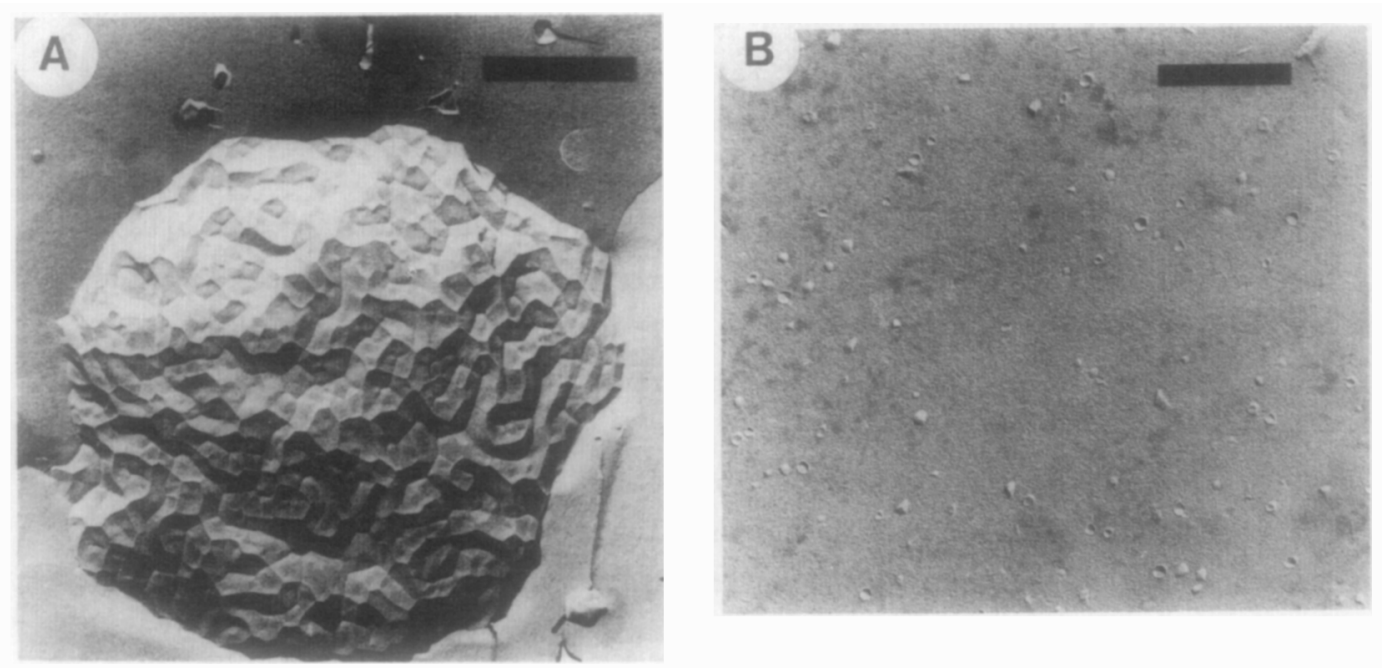

Figure 3. Freeze fracture electron micrographs of vortexed and sonfcated sulfatides

Sulfatide preparations were prepared, concentrated by centrifugation at $100,000 \times \mathrm{g}$ for $10 \mathrm{~min}$ and freeze fractured as described under Materials and Methods. Panel A shows a typical example of the particles found in a freeze fracture replica preparation of vortexed sulfatides at 30,000 times magnification. Sonlcated sulfatides were passed over Sepharose 2B and panel $B$ shows a freeze fracture replica obtalned from the material eluting from 20 to $24 \mathrm{ml}$ elurion volume at 43,000 magnificacion. Horizontal bars represent $3 \mathrm{~cm}$ in length. 
bllayers also displayed ordered corrugations similar to the corrugations that have been observed on fracture faces of synthetic lipids in the $P_{\alpha \beta}$ or $P_{\beta}$ phase [12-15]. It is 11kely, therefore, that at the temperature from which the sulfatide samples were quenched (room temperature) the lipid was in the solid gel state. This is supported by recent differential scanning calorinetric data which reported a phase transftion temperature of approximately $55^{\circ} \mathrm{C}$ for the transition of bovine braln sulfatides from the solid to the gel state [16]. It is also consistent with the fact that in order to obtain homogeneous suspensions, sulfatides have to be dispersed in buffer at temperatures well above $55^{\circ} \mathrm{C}$. Freeze fracture of sonicated sulfatides showed the existence of small vesicles (ranging in size from 400-2000 A diameter) and of stacked bilayer structures (data not shown). Fig. 3B shows a typical fracture face obtained from the material eluting in the Included peak (20-23 ml elution volume) showing sferical and flattened vesctles with a diameter around $500 \mathrm{~A}$.

In order to obtain sufficient amounts of particles for freeze fraction, the preparations were concentrated by centrifugation for $10 \mathrm{~min}$ at $100,000 x g$. Vortexed sulfatides spun down quantitatively. After sonication, however, approximately 70-75\% was recovered in the pellet. The vold volume peak could be completely recovered, whereas approximately $90 \%$ was recovered in the pellet from material eluting between 20-24 ml. Rechromatography of the resuspended pellets over Sepharose $2 B$ showed that no alteration in elution profile had occurred upon centrifugation. Therefore, the fracture faces obtained (FIg.3) are fully representative for vortexed preparations and for at least $90 \%$ of the sonlcated sulfatides eluting in the first half of the included peak.

The result that the majority of the 11p1d is present in the bilayer structure appears in contrast with earlier data reporting that sulfatides form micelles upon sontcation [17-20]. It is posstble that differences in sonication procedure (e.g. sonication time, intensity of sonication and temperature at which the confcation was performed) may account for these differences. In our study, upon sonication approximately 20-30\% of the sulfatides did not sediment upon centrifugation and eluted at the end of the Sepharose 2B column. When material eluting in the trailing edge of the peak (28-33 ml) was pooled and concentrated by dialysis in dialysis bags of cutoff MW 4000 against polyethylene glycol (20,000 MW) and subsequently subjected to freeze fracture a disproportional small amount of vestcles was found (data not shown). This indicates that upon sonication part of the sulfatides might be present as small non-centrifugable particles, possibly In the micellar form. Such particles will not be seen in the freeze fractures since structures of less than 100 A were not easily detectable with the technique eaployed. 
The data presented here indicate that the biologically active form of sulfatides responsible for the strong procoagulant activity is the bilayer structure. The vortexed preparations show large liposomes and blological activity associated with these 1iposomes. Under our conditions some $25 \%$ of sulfatides may be present as micelles in sonicated preparations. Since significant amounts of vesicles w111 be present, even in fractions eluting late in the column, we do not know as yet whether the sulfatide micelles also exert significant procoagulant activity. It is likely, however, that micelles are less potent in promoting contact activation since the activation of purified human Factor XII is strongly diminished with these particles (Tans, Rosing and Griffin, unpublished observations).

\section{REFERENCES}

1. Fujikawa, K., Hetmark, R.L., Kurachi, K. and Davie, E.W. (1980) Biochemistry $19,1322-1330$.

2. Tans, G. and Griffin, J.H. (1982) Blood 59, 69-75.

3. Contact phase coagulation disorders (1987) Semin. Thromb. Hemostas. 13 , no. 1 .

4. Karlsson, K.-A. (1982) In: Biological Membranes, vo1 4 (Chapman, D. Ed), pp. 1-74, Acad. Press, N.Y.

5. Kurachi, K., Fujikawa, K. and Davie, E.W. (1980) Biochemistry 19: 1330-1338.

6. Tans, G., Rosing, J. and Griffin, J.H. (1983) J. Biol. Chem. 258: 8215-8222.

7. Tans, G., Rosing, J., Berrettini, M., Lämmle, B., and Griffin, J.H. (1987) J. B1o1. Chem. 262, 11308 - 11314.

8. Rosing, J., Tans, G. and Griffin, J.H. (1985) Eur. J. Blochem. 151, 531-538.

9. Kean, E.L. (1968) J. Lipld Research 9, 319-327.

10. Branton, D. (1966) Proc. Nat1. Acad. Sci. USA 55, 1048-1056.

11. Moor, H. (1971) Phil. Trans. R. Soc. London. Sec. B. 261, 121-131.

12. Verkleif, A.J. and Ververgaert, P.H.J.TH. (1975) Ann. Rev. Phys. Chem. 26, 101-122.

13. Coste1lo, M.J. and Gulik-Krzywicki, T. (1976) Bioch1m. Blophys. Acta $455,412-432$.

14. Ranck, J.L., Mateu, L., Sadler, D.M., Tardleu, A., Gulik-Krzywicki, T. and Luzzatt1, V. (1974) J. Mo1. B1ol. 85, 249-277.

15. Gulik-Krzywick1, T. (1975) Bloch1m. Biophys. Acta 415, 1-28.

16. Koshy, K.M. and Boggs, J.M. (1983) Chem. Phys. Lip1ds 34, 41-53

17. Jeffrey, H.J. and Roy, A.B. (1977) Aust. J. Exp. Biol. Med. Sc1. 55: 339-346.

18. Gammack, D.B., Perkin, J.H. and Saunders, L. (1964) Biochim. Biophys. Acta 84, 576-586.

19. Cestaro, B., Pistoles1, E., Hershkowitz, H. and Gatt, S. (1982) Biochim. Biophys. Acta 685, 13-20.

20. Abrahamsson, S., Pasher, I, Larsson, K. and Karlsson, K. A. (1972) Chem. Phys. Lipids 8, 152-179. 